\title{
Identification of a non-stationary system using the Multi-Model approach
}

\author{
Khaled FAWAZ \\ Lebanese University, Faculty of Technology. Saïda. Lebanon.
}

\begin{abstract}
In this paper, we will present a real-time identification of a dynamic system with time-varying parameters: the Steam Generator (Precisely the Industrial Boiler), which is located at the LAGIS (Laboratory of Automation, Computer and Signal Engineering). ) of the Lille University. The approach used to identify the system is the Multimodel, which is an identification technique of Non-linear systems.
\end{abstract}

\section{Motivations}

A system is a set of interrelated objects or phenomena that are artificially isolated from the outside [1].

System modeling is often considered as the initial phase of the modern control. It allows determining the model of the process, which used to predict its future behavior, to diagnose its evolutions of operation or to synthesize its regulator and its control law. Two approaches are often used to model a system:

The first is called "white box modeling" or "theoretical modeling". It is obtained from a priori knowledge of the system, including laws governing physical, chemical, mechanical, biochemical, occurring in the system.

The second approach known as "black box modeling" which makes it possible, from experimental data, to establish a relationship between the inputs and outputs of the system, and does not require a priori knowledge of the physical laws.

\section{Introduction: Multi-Model Approach}

Multi-Model approach consists in representing a non-linear system by a set of simple local models (linear or non-linear models) valid in certain areas of system operation. The overall model of the system is an interpolation of local models through validity functions associated with these models (Fig.1).

In recent years the multi-model approach has found a certain interest: [2], [3].

Most identification methods based on the multi-model approach are proposed for MISO (Multi-Input, Single-Output) systems. In the case where the system is MIMO, they assume that the outputs are decoupled and the system can be decomposed into a MISO subsystem. In our study, we based on the work proposed in [4] and adapted to the MIMO system. 
We will first describe the problem of identification that will be addressed by the multimodel approach, then describe the principles and the different stages of this method. We will limit our study to discrete time dynamic systems.
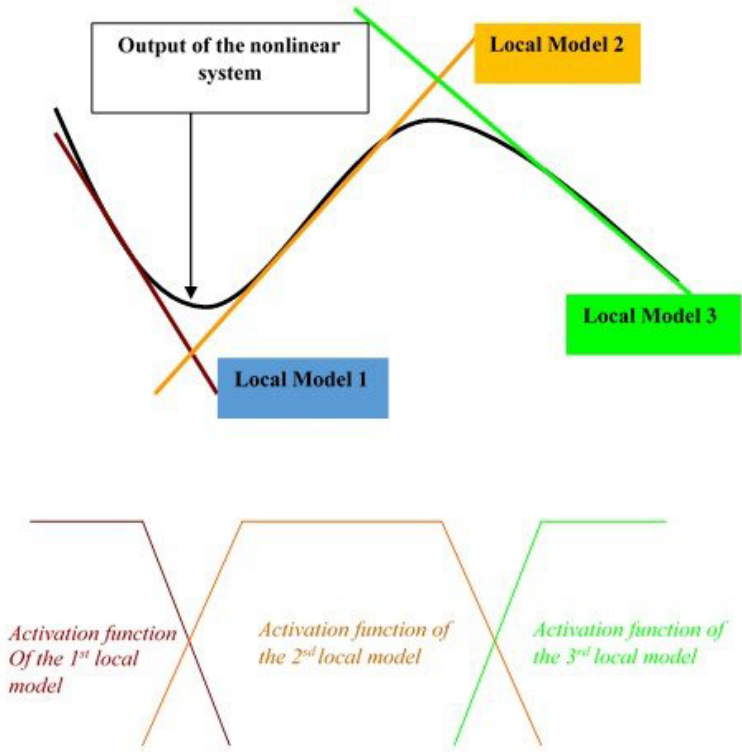

Fig.1:Multi-Model Approach

\section{Approach Description}

We will present in this section the multi-model approach and define the identification problem to be treated. [5], [6].

Consider the non-linear system given by the following input/output model:

$$
y(t)=F(\varphi(t))
$$

Where $\varphi(t)$ is the regression vector.

Suppose we have $P$ local models $f_{i}(\varphi(t))$ that characterize the system in different areas of operation. Each local model is associated with an activation function $\rho_{i}\left(\varphi(t), \alpha_{i}\right)$ parameterized by the vector $\alpha_{i}$ such as the global model (1) can be represented as a combination of local models:

$$
y(t)=\frac{\sum_{i=1}^{P} \rho_{i}\left(\varphi(t), \alpha_{i}\right) f_{i}(\varphi(t)}{\sum_{j=1}^{P} \rho_{j}\left(\varphi(t), \alpha_{j}\right)}, \text { let } W_{i}(\varphi(t), \alpha)=\frac{\sum_{i=1}^{P} \rho_{i}\left(\varphi(t), \alpha_{i}\right)}{\sum_{j=1}^{P} \rho_{j}\left(\varphi(t), \alpha_{j}\right)}
$$

We obtain the general expression of a multi-model structure:

$$
Y(t)=\sum_{i=1}^{P} W_{i}\left(\varphi(t), \alpha_{i}\right) f_{i}(\varphi(t))
$$


The local model can be written as:

$$
y_{i}(t)=\theta_{i} \varphi_{i}(t)
$$

Where $\theta_{i}$ is the parameter vector of a local model. The output of the global model becomes

$$
y(t)=\sum_{i=1}^{P} W_{i}\left(\varphi(t), \alpha_{i}\right) y_{i}(t)
$$

We will limit to the case where the multi-model is with equation error, the regression vector is obtained by the inputs and the outputs of the time shifted system.

$$
\varphi_{i}(t)=\left[\begin{array}{c}
y(t-n) \\
\vdots \\
y(t-1) \\
u(t-m) \\
\vdots \\
u(t-1)
\end{array}\right]
$$

Later, the operating space will no longer be the regression space, but a space $\mathrm{Z}$ generated by the vector. The components of this vector are called the characteristic variables. They are either inputs or outputs or other variables that take into account the non-linearity of the system.

Local functions and activation functions are now depend on these characteristic variables.

The overall output of the system becomes:

$$
y(t)=\sum_{i=1}^{P} y_{i}(t) W(z(t))
$$

Where $z(t)$ the characteristic variable and $W$ is the activation function of the local model.

Equation (4) is rewritten by multiplying the outputs by $W_{i}$; we obtain by stacking on a horizon $j$ :

$\left[y_{i}(t-j+1) W\left(z_{(t-j+1)}\right)\left|y_{i}(t-j+2) W\left(z_{(t-j+2)}\right)\right| \cdots \mid y_{i}(t) W\left(z_{(t)}\right)\right]=\theta_{i}\left[\varphi_{i}(t-j+1) W\left(z_{(t-j+1)}\right)|\cdots| \varphi_{i}(t) W\left(z_{(t)}\right)\right]$

Which is written as:

$$
\left[y_{i}(t-j+1) W\left(z_{(t-j+1)}\right)\left|y_{i}(t-j+2) W\left(z_{(t-j+2)}\right)\right| \cdots \mid y_{i}(t) W\left(z_{(t)}\right)\right]=\theta_{i} \Phi \Omega_{i}
$$

Where:

$$
\Phi=\left[\varphi_{i}(t-j+1)\left|\varphi_{i}(t-j+2)\right| \cdots \mid \varphi_{i}(t)\right]
$$




$$
\Phi=\left[\begin{array}{cccc}
y(t-j+1-n) & y(t-j+2-n) & \cdots & y(t-n) \\
\vdots & \vdots & \vdots \\
y(t-j-1) & y(t-j) & y(t-2) \\
y(t-j) & y(t-j+1) & y(t-1) \\
z u(t-j+1-n) & u(t-j+2-n) & u(t-n) \\
\vdots & \vdots & \vdots \\
z(t-j) & u(t-j+1) & u(t-1) \\
u(t-j+1) & u(t-j+2) & u(t)
\end{array}\right] \quad \Omega_{i}=\left[\begin{array}{cccc}
W\left(z_{(t-j+1)}\right) & 0 & \ldots & 0 \\
0 & W\left(z_{(t-j+1)}\right) & 0 \\
\vdots & & \ddots & \vdots \\
0 & & & W\left(z_{(t)}\right)
\end{array}\right]
$$

Hankel Matrices (10)

Equation (8) allows us to write:

$\sum_{i=1}^{P}\left[y_{i}(t-j+1) W\left(z_{(t-j+1)}\right)\left|y_{i}(t-j+2) W\left(z_{(t-j+2)}\right)\right| \cdots \mid y_{i}(t) W\left(z_{(t)}\right)\right]=\sum_{i=1}^{P} \theta_{i} \Phi \Omega_{i}$

In addition, according to (7)):

$$
\sum_{i=1}^{P}\left[y_{i}(t-j+1) W\left(z_{(t-j+1)}\right)\left|y_{i}(t-j+2) W\left(z_{(t-j+2)}\right)\right| \cdots \mid y_{i}(t) W\left(z_{(t)}\right)\right]=[y(t-j+1) \cdots y(t)]
$$

Which give:

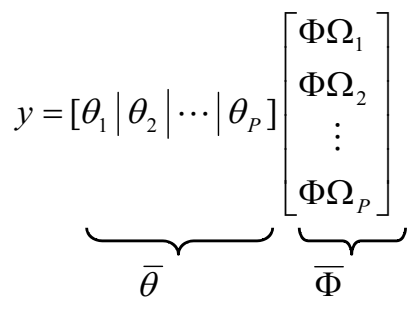

Finally, the estimation of the parameters vector is given by:

$$
\bar{\theta}=y(\bar{\Phi})^{\perp}
$$

Where $(\bar{\Phi})^{\perp}$ is the pseudo-inverse of $\bar{\Phi}$.

\section{Boiler identification}

We will apply the identification method based on the multi-model approach to identify the system. This application involves the determination of an activation variable that determines the non-linearity of the system. The number of local models must also be determined as well as the order of these local models. The parameters of the local models will then be estimated by the least squares as well as the activation functions that determine the validity of each local model. Two applications will be made in the following: the first concerns a MISO subsystem of the boiler while the second concerns the entire MIMO system.

Characteristics: Heat output: $60 \mathrm{~kW}$; Pressure: 10 bars; Steam production: $87 \mathrm{~kg} / \mathrm{h}$; Power supply: three-phase $380 \mathrm{~V}$; Volume: 175 liters. 


\subsection{Determining of the characteristic variables}

The characteristic variables (or activation variables) are inputs, either outputs or other variables that take into account the non-linearity of the system.

It can be seen from the results obtained in Fig.2 that there is a strong collinearity between the pressure P7 (Pressure inside the boiler) and temperatures T5 (Water temperature inside the boiler) and T6 (Steam temperature inside the boiler). The level L8 (Water level in the boiler) has saturation effects. Our choice was therefore the pressure P7 as a characteristic variable because it presents the effect of the non-linearity of the system; it is also in direct relation with the other variables.

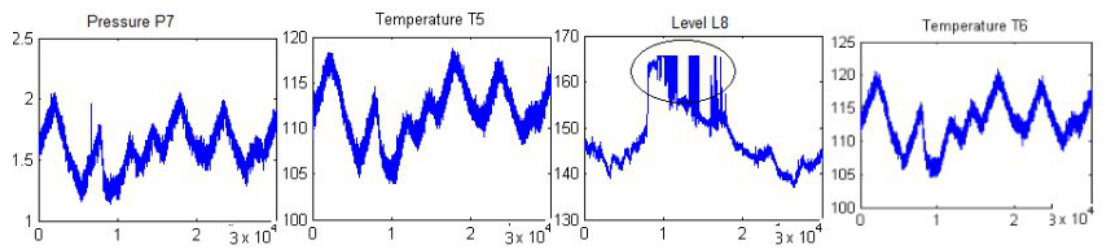

Fig.2: Determining of the charecteristic variables

\subsection{Number of Local models}

The strategy we adopted in order to determine the number of local models consist of starting with a single model and gradually adding to the satisfaction of the criterion of stop. We chose two local models for the application (prediction of the pressure). Activation functions are obtained from the sigmoid, and they cover the entire range of variation of the characteristic variable, which varies between 1 and 10 bar.

\subsection{Model order}

For simplicity reason, we assumed that all local linear models have the same orders. The order of the local models (output and input) have been set to 2 . By observing the prediction error $\sum_{k=1}^{N}\left(y_{k}-\hat{y}_{k}\right)^{2}$ (where $\mathrm{N}$ is the total number of data) according to the system order, we have noticed that beyond 2, the error no longer decreases (it decreases in a negligible way), while the complexity of the model increases. 


\subsection{Identification Algorithm}

1) Initialization:

Algorithm 1:

a. Determination of the number of local models no.

b. initialization of the distance vector $d_{i}$ between activation functions

c. Choice of the characteristic variables $Z(t)$ and of the interval [zmin, zmax], range of variation of the characteristic variables

d. Set parameter $\gamma$ : slope of activation functions

2) Estimates of the model parameters $\theta_{i}$ from the activation functions obtained by $d_{i}$

3) Estimates of $d_{i}$ (non-linear function of Matlab)

4) Performance verification:

if the model is satisfactory then close if not back to step 2

\section{Application: Boiler identification}

Consider the following subsystem with three inputs and one output:

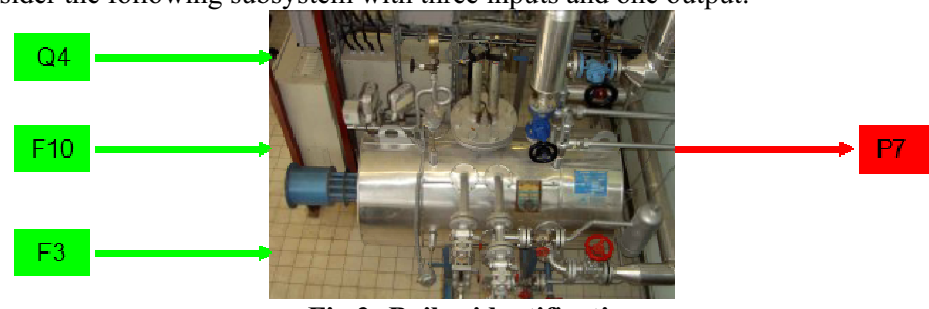

Fig.3: Boiler identification

The input-output data used to identify this system are shown in Fig.4. The data are collected from the actual system over a period of 6200 seconds. The sampling period is $1 \mathrm{~s}$.
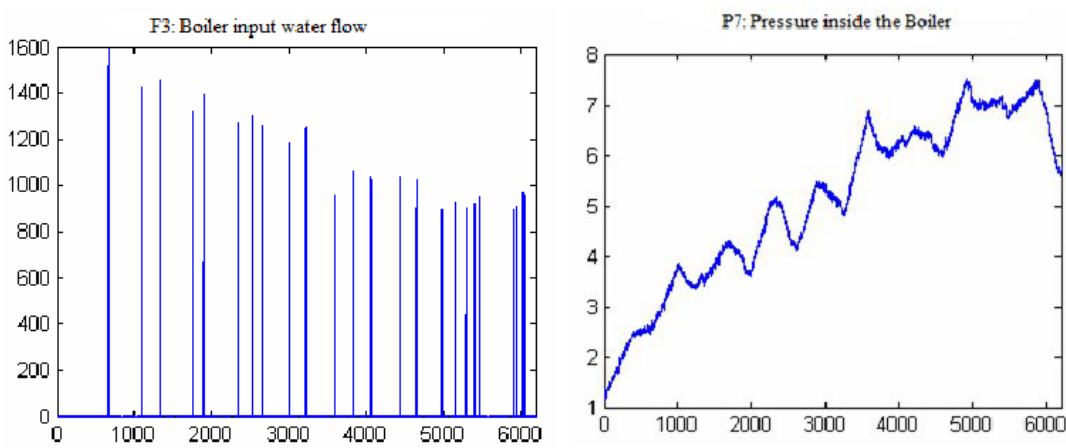

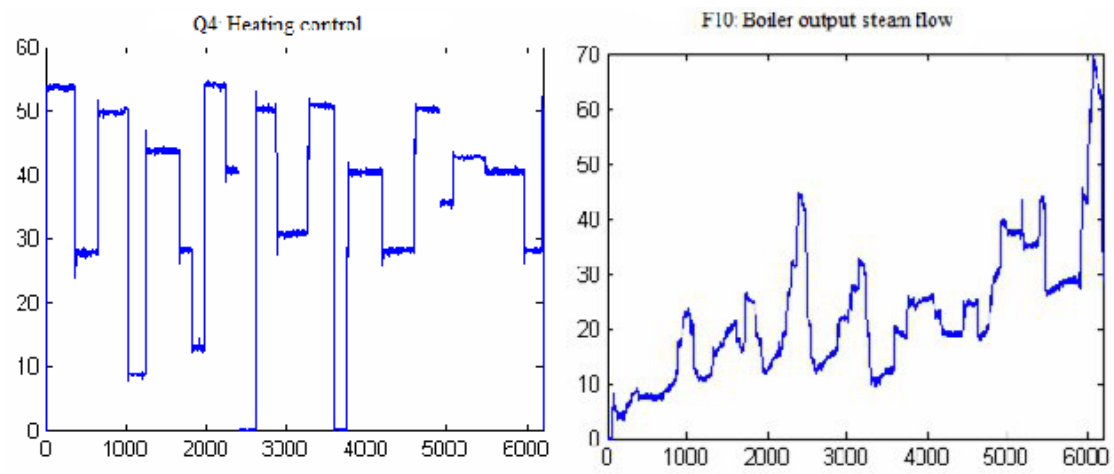

Fig:4: Inputs-Outputs data used for system identification

Two local models were used in this case, (the local models are in input/output form, the regression vector is formed by the inputs and outputs of the system shifted in time). These models are represented in Fig.5.
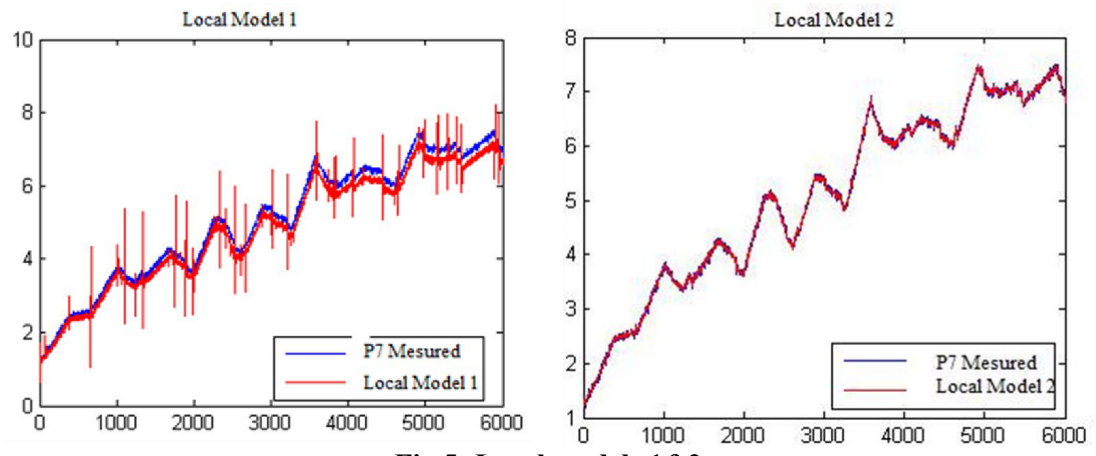

Fig.5: Local models 1\&2

Fig.5 shows the weighted output of each local linear model. Note that the first model is affected by noise. This is because the output is not filtered. These two local models are activated by two validity functions plotted over the entire variation range of P7 (Fig.6).

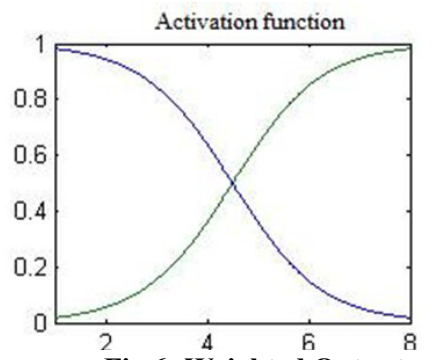

Fig.6: Weighted Outputs

The global model has been validated with a set of data different from the one used for the calculation of the model. 
We then opted for a real-time identification of the pressure P7, the model was implanted under Simulink. The results of online identification are given in Fig.7.
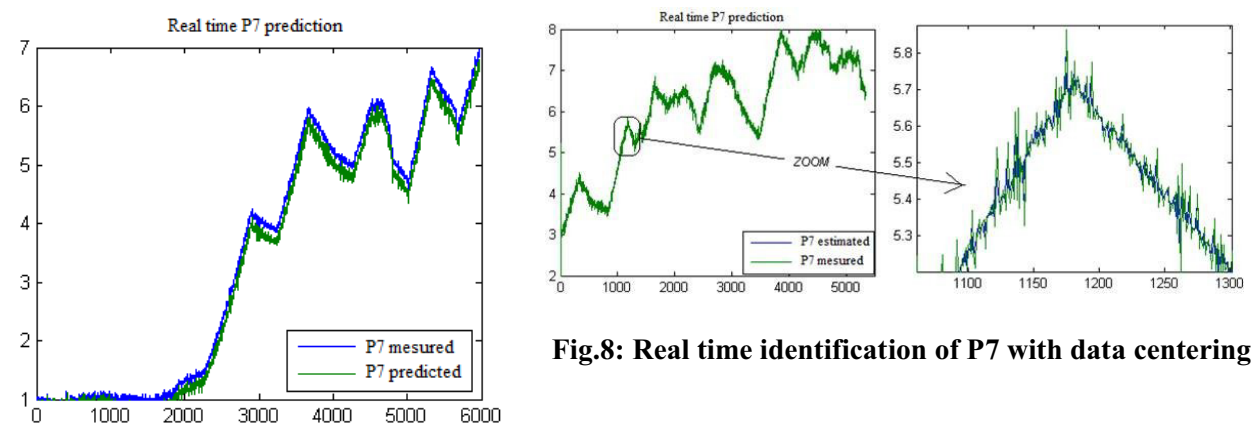

Fig.8: Real time identification of $P 7$ with data centering

Fig.7: Real time identification of P7

The first result shows that the pressure estimate is perfect. There is a small offset between the 2 outputs (estimated and measured), this is due to the fact that the data are not centered. We therefore refocused the data $(\mathrm{P} 7=$ characteristic variable) and identified the model again. The performance of the new model obtained is shown in Fig 8.

\section{Conclusion}

We presented in this work a real-time identification application of a dynamic system (industrial boiler) with time-varying parameters using the multi-model approach. The results were very conclusive, and very satisfactory. We should note the importance of data processing before applying the identification procedures, and the importance of data centering for calculating the local models. The approach used (multi-model) has shown great reliability and robustness despite the effect of noise and the strong variation of the dynamics of the system

\section{References}

1. L. Ljung. System Identification, Theory of the User.

2. K. Gasso. Identification des systèmes dynamiques non-linéaires : approche multimodèle. thèse de doctorat 2000.

3. A. Boukhris, G. Mourot \& J. Ragot (1999) Non-linear dynamic system identification: A multi-model approach, International Journal of Control, 72:7-8, 591-604, DOI: 10.1080/002071799220795

4. K. Pekpe. Identification par les techniques des sous-espaces - application au diagnostic. These de doctorat 2004.

5. K. Midzodzi Pekpe, G. Mourot, K. Gasso, J. Ragot. Identification d'une représentation nmultimodèle des systèmes dynamiques non linéaires par la méthode des sous-espaces. Journées Doctorales d'Automatique, JDA'03, Jun 2003, Valenciennes, France.

6. Gasso K., Gilles M., and Ragot J., Structure identification in multiple model representation: elimination and merging of local models. Proceedings of the 40th IEEE Conference onDecision and Control, pp. 2992-2997, Orlando,Florida USA, December 2001. 